\title{
Papers
}

\section{Neurological sequelae in twins born after assisted conception: controlled national cohort study}

Anja Pinborg, Anne Loft, Lone Schmidt, Gorm Greisen, Steen Rasmussen, Anders Nyboe Andersen

\begin{abstract}
Objective To compare neurological sequelae in twins born after assisted conception with singletons after assisted conception and naturally conceived twins and to assess neurological sequelae in children conceived after in vitro fertilisation (IVF) compared with intracytoplasmic sperm injection (ICSI).
\end{abstract}

Design Controlled, national register based, cohort study. Participants Twins $(\mathrm{n}=3393)$ and singletons $(\mathrm{n}=5130)$ conceived by using assisted reproductive technologies and naturally conceived twins ( $\mathrm{n}=10239)$ born in Denmark between 1995 and 2000. The children's age at time of follow up was 2-7 years.

Data sources Children were identified by cross linkage of the national medical birth registry and the national registry for in vitro fertilisation. Neurological and psychiatric diagnoses were retrieved from the national patients' registry and the Danish psychiatric central registry.

Main outcome measures Neurological sequelae, defined as cerebral palsy, mental retardation, severe mental developmental disturbances, and retarded psychomotor development. Further we made separate analyses on the specific cerebral palsy diagnosis.

Results The crude prevalence rates per 1000 of neurological sequelae in twins and singletons after assisted conception and in naturally conceived twins were 8.8, 8.2, and 9.6, and of cerebral palsy 3.2, 2.5, and 4.0, respectively. In twins after assisted conception compared with control twins, the odds ratios of neurological sequelae and specifically of cerebral palsy, adjusted for child sex and year of birth, were 0.9 (95\% confidence interval 0.6 to 1.4 ) and 0.8 (0.4 to 1.6), respectively. The corresponding odds ratios for twins after assisted conception compared with singletons after assisted conception were 1.1 ( 0.7 to 1.7$)$ for neurological sequelae and 1.3 (0.6 to 2.9) for cerebral palsy. The odds ratio of neurological sequelae in children conceived by ICSI was 0.9 (0.5 to 1.7$) v$ children conceived by IVF.

Conclusions Twins from assisted conception have a similar risk of neurological sequelae as their naturally conceived peers and singletons from assisted conception. Children born after ICSI have the same risk of neurological sequelae as children born after IVF.

\section{Introduction}

In Denmark 5\% of infants are the result of in vitro fertilisation (IVF) techniques (IVF and intracytoplasmic sperm injection, ICSI) , and the latest European data from 2000 showed that 39\% of IVF infants were born as twins. ${ }^{1}$ Hence assisted conception and increasing maternal age have had a great impact on the national twin birth rates.

Several studies have shown that twin pregnancies are the main reason for the overall poorer neonatal outcome in pregnancies after assisted conception. ${ }^{2-5}$

In population based studies on naturally conceived children, twins have four times the risk of cerebral palsy as singletons . However, the literature specifically addressing long term morbidity in twins after assisted conception is limited. A Swedish register study has found an increased risk of cerebral palsy in children after assisted conception, mainly because of the high rate of twins. ${ }^{7}$ Our recent Danish questionnaire study showed similar morbidity in twins after assisted conception and naturally conceived twins, but compared with singletons after assisted conception twins were more likely to have surgical interventions, special needs, and delayed speech development, whereas the prevalence of neurological sequelae was equal. ${ }^{8}$

To study the long term effects of IVF techniques on twins we established a database with all singletons and twins born after assisted conception between 1995 and 2000. Perinatal outcomes including prevalence rates of malformations, malignancies, mortality, and data on the use of treatments for special needs, including speech therapy, in these children have been published recently. ${ }^{8-10}$

We assessed prevalence rates of neurological sequelae in Denmark in a nationwide cohort of twins after IVF techniques and in two population based control groups of naturally conceived twins and of singletons conceived by IVF techniques. We also compared the roles of ICSI and conventional IVF in neurological sequelae in these children.

\section{Methods}

\section{Participants}

We used the Danish medical birth registry recording all births in Denmark to identify all women giving birth to twins and singletons from 1 January 1995 to 31 December 2000. ${ }^{11}$ All citizens in Denmark have a unique identification number in the civil registration system. Identification of women in the birth registry was based on this number. Subsequently, a cross reference with the Danish registry for in vitro fertilisation enabled us to dichotomise into women who conceived naturally or after IVF. We used a unique existing linkage between the identification number of a mother in the civil registration system and her children in the medical birth registry to establish the identification number of every individual child in the three cohorts. We drew records on fertilisation method and obstetric outcome from the compulsory IVF registry and the medical birth registry. ${ }^{11}$ 


\section{Outcome measures}

We cross referenced the Danish patients' registry to identify all children diagnosed or treated in a hospital setting from birth until 31 December 2002. ${ }^{13}$ We classified diagnoses in the patients' registry according to the International Classification of Diseases, 10th edition (ICD-10). As diagnoses in relation to a psychiatric hospital setting are handled with tight control in Denmark, we drew these diagnoses from Denmark's psychiatric central registry. ${ }^{14}$

We established a database with files from the different registries with each individual child in the three cohorts as the key variable and entered the neurological and psychiatric diagnoses as outcomes. To ensure an accurate neurological diagnosis, all children were aged between 2 years and 7 years at time of follow up (31 December 2002). We included the following ICD-10 diagnosis codes on inpatients and outpatients from the patients' registry and the psychiatric registry as neurological sequelae: mental retardation (DF70.0-79.9), severe mental developmental disturbances (DF84.0-89.9), cerebral palsy (DG80.0-83.8), and retarded psychomotor development (DR62.0). We performed separate analyses on the specific diagnosis cerebral palsy as this is a well defined diagnostic entity. This made our study comparable to previous studies on the same subject, which also dealt with cerebral palsy separately. We allowed each child to be counted only once with a diagnosis code in each of the three main diagnosis groups (DF, DG, and DR). If a child had more than one diagnosis in the same main diagnosis group, we gave priority in the following order: severity of the diagnosis, specificity, and latest recorded diagnosis. We ranked diseases arbitrarily from 1 to 10 ( 1 with highest severity) before retrieving data; the ranking is available from AP. We did not include stillborn children but included children dying from delivery until 31 December 2000 in the cohorts. Since 31 December 2000 the Danish registry of causes of deaths has not been updated.

\section{Statistical analysis}

We used SPSS for Windows, version 10.0, for our statistical analysis. We considered a probability value of $\mathrm{P}<0.05$ statistically significant. We used Student's $t$ test to analyse differences of means of continuous parametric data and $\chi^{2}$ tests to compare distributions between groups. We used the Mantel-Haenszel estimate after stratification for a child's sex and year of birth to calculate odds ratios. In all analyses we analysed twins as separate children and not as a pair.

We performed two separate multiple logistic regression analyses to determine which variables independently predicted the dichotomous outcomes of neurological sequelae or not and of cerebral palsy or not. In all regression analyses we used the primary diagnosis of each child as the outcome variable. We entered the following variables as potential confounders: mother's age at delivery ( $<35$ years or $\geq 35$ years), sex of child, year of birth, IVF or ICSI, low birth weight $(<2500 \mathrm{~g})$, and low gestational age $(<37$ weeks). We performed three separate analyses, one for all three cohorts, a second for twins alone (IVF or ICSI and controls), and a third restricted to children conceived by IVF or ICSI, both singletons and twins.

\section{Results}

\section{Demographic data}

We included 3393 twins conceived by IVF or ICSI, 10239 naturally conceived twins, and 5130 IVF or ICSI singletons in the study. Table 1 shows mothers' and infants' characteristics. Since 41 IVF twins and 95 control twins were survivors of a stillborn co-twin, the number of children in both twin cohorts was odd. As expected, mothers of IVF or ICSI twins were older than control twin mothers but younger than mothers of IVF or ICSI singletons. For 1676 IVF-ICSI twin pairs and for 5103 control twin pairs the sex of both twins was known. In contrast to $65.3 \%$ $(3330 / 5103)$ control twin pairs with the same sex, only $50.8 \%$ $(851 / 1676)$ of the IVF or ICSI twins were same sex $(\mathrm{P}<0.001$; table 1). The zygosity of twins can be determined by Weinberg's differential method. ${ }^{15}$ In our study, this estimation results in $1.6 \%$ (26/1676) monozygotic IVF or ICSI and 31\% (1557/5103) monozygotic control twin pairs. We adjusted all analyses for year of birth to account for the differences in average child age at time of follow up.

\section{Neurological sequelae}

We observed similar prevalence rates of neurological sequelae in IVF or ICSI twins and the two control groups. The crude prevalence of children with neurological sequelae was 8.8/1000 in IVF or ICSI twins, 9.6/1000 in control twins, and 8.2/1000 in IVF or ICSI singletons. The prevalence rates of the specific diagnoses cerebral palsy and mental retardation were also similar (table 2). Odds ratios of neurological sequelae, cerebral palsy, and mental retardation with and without adjustment for a child's sex and year of birth were the same in IVF or ICSI twins and both control groups (table 3). The odds ratios of neurological sequelae in ICSI compared with IVF children were 1.3 (95\% confidence interval 0.6 to 3.0) for twins and 0.5 (0.2 to 1.2) singletons.

We identified one twin pair with neurological sequelae in 30 IVF or ICSI twins and six in 98 control twins, all of the same sex. The concordance rates were therefore $3.3 \%$ and $6.1 \%$, respectively.

\section{The role of zygosity}

To account for the higher monozygotic rate among naturally conceived twins, we computed odds ratios of neurological sequelae in IVF or ICSI twins of the opposite sex compared with twins of the same sex $(0.7,0.3$ to 1.4$)$ and in control twins (1.0, 0.8 to 1.4).

To exclude the monozygotic twins we restricted our analyses to twins of the opposite sex. The odds ratios for neurological sequelae were 1.1 (0.6 to 2.3), mental retardation 1.1 (0.4 to 2.6), and cerebral palsy 1.3 ( 0.4 to 4.0$)$ in twins of the opposite sex conceived by IVF or ICSI compared with control twins of the opposite sex.

\section{Factors influencing the risk of neurological sequelae}

We performed multiple logistic regression analyses for all children in the three cohorts and for twins alone to explore the effect of relevant confounders on the risk of neurological sequelae. In each of the analyses we tested separately the risk of the two outcomes, neurological sequelae and cerebral palsy. Table 4 shows that low birth weight or prematurity and male sex were strong risk factors for both outcome measures. After adjustment for low birth weight or prematurity, we observed that IVF and maternal age $>35$ years had no independent effect on the risk of neurological sequelae, and neither had being a twin. In the analyses restricted to twins, IVF or ICSI twins had no greater risk of neurological sequelae than naturally conceived twins (table 4). We adjusted all data in the logistic regression analysis for children's year of birth.

To study the effect of ICSI, we performed regression analyses restricted to IVF or ICSI children. ICSI children had similar odds ratios as IVF children for neurological sequelae $(0.9,0.5$ to 1.6) and cerebral palsy $(0.8,0.3$ to 2.4$)$. Also in these analyses male sex and low birth weight or prematurity independently affected the 


\begin{tabular}{|c|c|c|c|c|c|}
\hline & IVF-ICSI twins & Control twins & P value* & IVF-ICSI singletons & P value \\
\hline No of babies born alive & 3393 & 10239 & & 5130 & \\
\hline No of stillborn babiesł & $45(13.1 / 1000)$ & $123(11.8 / 1000)$ & 0.6 & $34(6.6 / 1000)$ & 0.002 \\
\hline $\begin{array}{l}\text { Mean children's age at follow up in years } \\
\text { (SD) }\end{array}$ & $4.2(1.7)$ & $4.4(1.7)$ & $<0.001$ & $4.1(1.7)$ & 0.12 \\
\hline \multicolumn{6}{|l|}{ Sex of babies:§ } \\
\hline No $(\%)$ of boys & $1747 / 3352(52.1)$ & $5237 / 10215(51.3)$ & 0.4 & $2700(52.6)$ & 0.6 \\
\hline No $(\%)$ of girls & $1605 / 3352(47.9)$ & $4978 / 10215(48.7)$ & & $2430(47.4)$ & \\
\hline No $(\%)$ of twin pairs of the same sex & $851 / 1676(50.8)$ & $3330 / 5103(65.4)$ & $<0.001$ & - & \\
\hline Mean birth weight in g (SD) & $2508(615)$ & $2540(612)$ & 0.01 & $3457(629)$ & $<0.001$ \\
\hline Mean gestational age in weeks (SD) & $35.9(3.0)$ & $36.1(2.9)$ & 0.02 & $39.3(2.2)$ & $<0.001$ \\
\hline \multicolumn{6}{|l|}{ No of deaths per 1000 babiesf: } \\
\hline Early neonatal, up to day 6 & $26(7.6)$ & $119(11.6)$ & 0.05 & $23(4.5)$ & 0.06 \\
\hline Late neonatal, day 7-28 & $4(1.2)$ & $22(2.1)$ & 0.3 & $3(0.6)$ & 0.4 \\
\hline Day 29-1 year & $5(1.5)$ & $13(1.3)$ & 0.8 & $11(2.1)$ & 0.5 \\
\hline$>1$ year & $1(0.3)$ & $4(0.4)$ & & 0 & \\
\hline Total infant deaths & $36(10.6)$ & $158(15.4)$ & 0.04 & $37(7.2)$ & 0.1 \\
\hline $\begin{array}{l}\text { Mean maternal age at delivery in years } \\
\text { (SD) }\end{array}$ & $33.1(3.7)$ & $30.5(4.5)$ & $<0.001$ & $33.8(3.7)$ & 0.001 \\
\hline No $(\%)$ of mothers aged $<30$ years & $333 / 1668(20.0)$ & $2326 / 5119(45.4)$ & $<0.001$ & $791 / 5128(15.4)$ & $<0.001$ \\
\hline $\begin{array}{l}\text { Odds ratio (95\% confidence interval) of } \\
\text { mothers aged }<30 \text { years } v \text { mothers }>30 \\
\text { years }\end{array}$ & 1 & 3.4 (2.9 to 3.8$)$ & $<0.001$ & 0.7 (0.6 to 0.8$)$ & $<0.001$ \\
\hline \multicolumn{6}{|l|}{ No (\%) of women treated with: } \\
\hline In vitro fertilisation & $1262 / 1673(75.4)$ & - & & $3456 / 4605(75.0)$ & 0.8 \\
\hline Intracytoplasmic sperm injection & $411 / 1673$ (24.6) & - & & $1149 / 4605(25.0)$ & \\
\hline
\end{tabular}

*Differences of means of continuous parametric data were analysed with the use of Student's $t$ test.

†Distributions between groups were compared by using Pearson's $\chi^{2}$ analyses.

‡Stillborn children were not included in the cohorts and analyses.

§The sex was known for 3352 IVF or ICSI twins, 10215 control twins, and 5130 IVF or ICSI singletons.

ๆChildren, who died were registered until 31 December 2000. After that point data from the Danish registry of causes of deaths were not updated. Children who died after delivery were included in the cohorts.

risk of both outcomes, and we found no difference between twins and singletons in the risk of any of the outcomes.

Table 2 Numbers of children in the three cohorts with neurological sequelae. Diagnoses were counted per child. The primary diagnoses were classified according to severity in the ranking order system. Diagnoses were recorded from 1 January 1995 to 31 December 2002

\begin{tabular}{|c|c|c|c|c|c|}
\hline Neurological sequelae & IVF-ICSI twins ( $\mathrm{n}=3393$ ) & Control twins ( $n=10239$ ) & $\begin{array}{c}\text { P value (Pearson's } \chi^{2} \\
\text { test) }\end{array}$ & IVF-ICSI singletons $(n=5130)$ & $\begin{array}{c}\text { P value (Pearson's } \chi^{2} \\
\text { test) }\end{array}$ \\
\hline Mental retardation & 4 & 14 & 0.8 & 9 & 0.5 \\
\hline Infantile autism & 3 & 8 & 0.9 & 8 & 0.4 \\
\hline Asperger's syndrome & - & 3 & 0.4 & - & - \\
\hline Retarded psychomotor development & 12 & 32 & 0.7 & 12 & 0.3 \\
\hline Cerebral palsy & 11 & 41 & 0.5 & 13 & 0.5 \\
\hline Total & $30(8.8 / 1000)$ & $98(9.6 / 1000)$ & 0.7 & $42(8.2 / 1000)$ & 0.7 \\
\hline
\end{tabular}

Neurological sequelae were defined as mental retardation and severe mental developmental disturbances (DF70.0-DF79.9, DF 84.0-DF89.9) including infantile autism (DF84.0) and Asperger's syndrome (DF84.5), cerebral palsy (DG80.0-DG83.8), and retarded psychomotor development (DR62.0).

Table 3 Odds ratio of neurological sequelae, cerebral palsy and mental retardation in IVF-ICSI twins versus control twins and IVF-ICSI singletons. Results are presented as odds ratios and adjusted odds ratios for child sex and year of birth with $95 \%$ confidence intervals

No of children

No of children with disorders

Odds ratio $(95 \% \mathrm{Cl})$

Adjusted odds ratio $(95 \% \mathrm{CI})^{*}$

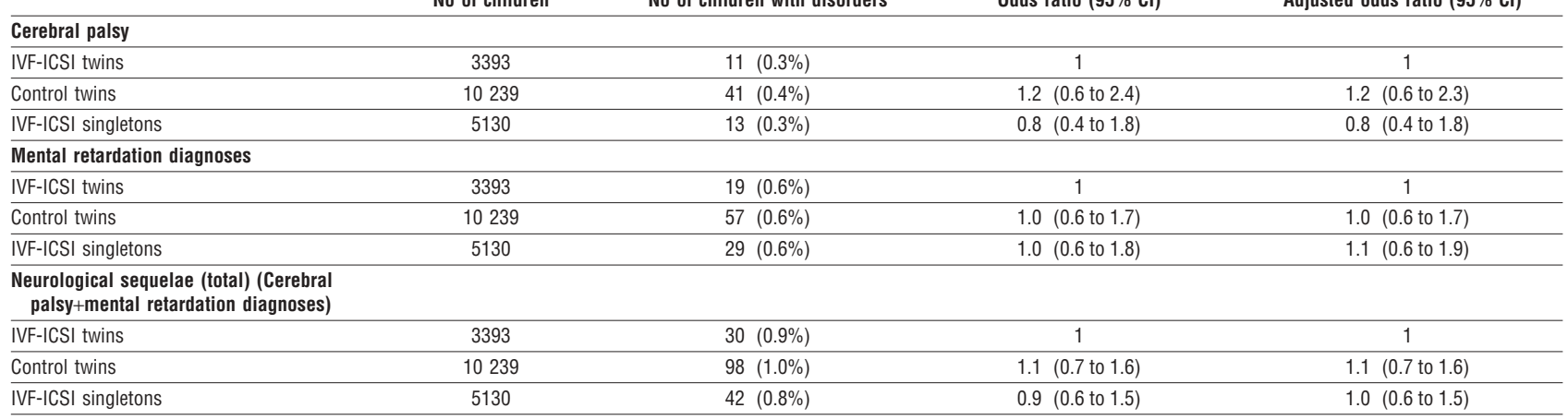

${ }^{*}$ Adjusted for child's sex and year of birth. 
Table 4 Multiple logistic regression analysis showing independent effects of being a twin infant, assisted conception (IVF and ICSI), maternal age $\geq 35$ years, male sex, and low birth weight $(<2500 \mathrm{~g})$ (upper panel) or low gestational age ( $<37$ weeks) (lower panel) on the risk of neurological sequelae and cerebral palsy. Results are presented as odds ratios (95\% confidence intervals), adjusted for child sex and year of birth

\begin{tabular}{|c|c|c|c|c|}
\hline & \multicolumn{2}{|c|}{ Neurological sequelae } & \multicolumn{2}{|c|}{ Cerebral palsy } \\
\hline & All cases & Twins & All cases & Twins \\
\hline \multicolumn{5}{|l|}{ Effects of birth weight } \\
\hline Twin & 0.7 (0.4 to 1.2$)$ & - & $0.6(0.2$ to 1.4$)$ & - \\
\hline Assisted conception & 0.9 (0.6 to 1.4) & 0.9 (0.6 to 1.4) & 0.8 (0.4 to 1.5) & 0.8 (0.4 to 1.5$)$ \\
\hline Low birth weight & 2.3 (1.6 to 3.2$)$ & 1.9 (1.4 to 2.8$)$ & 4.4 (2.5 to 7.8$)$ & $3.0(1.7$ to 5.4$)$ \\
\hline Male sex & 2.0 (1.4 to 2.8$)$ & 1.9 (1.3 to 2.8$)$ & 1.9 (1.1 to 3.2$)$ & 2.0 (1.1 to 3.5$)$ \\
\hline Maternal age $\geq 35$ years & 0.8 (0.6 to 1.2$)$ & 0.8 (0.5 to 1.4$)$ & 0.8 (0.4 to 1.5$)$ & $0.8(0.4$ to 1.7$)$ \\
\hline \multicolumn{5}{|l|}{ Effects of gestational age } \\
\hline Twin & 0.8 (0.5 to 1.3) & - & $0.6(0.2$ to 1.4$)$ & - \\
\hline Assisted conception & 0.9 (0.6 to 1.4$)$ & 0.9 (0.6 to 1.4) & 0.8 (0.4 to 1.6$)$ & $0.8 \quad(0.4$ to 1.6$)$ \\
\hline Low gestational age & 2.0 (1.4 to 2.8$)$ & 1.8 (1.2 to 2.6$)$ & 4.5 (2.5 to 8.1$)$ & 3.1 (1.7 to 5.7$)$ \\
\hline Male sex & 1.9 (1.3 to 2.6$)$ & 1.8 (1.2 to 2.6$)$ & $1.8(1.0$ to 3.0$)$ & 1.9 (1.0 to 3.4$)$ \\
\hline Maternal age $\geq 35$ years & 0.8 (0.6 to 1.2$)$ & $0.8(0.5$ to 1.4$)$ & 0.8 (0.4 to 1.5$)$ & 0.8 (0.4 to 1.8$)$ \\
\hline
\end{tabular}

\section{Discussion}

Twins born after assisted conception have a similar risk of neurological sequelae as naturally conceived twins and singletons after IVF or ICSI. The risk of neurological sequelae in children born after ICSI and conventional IVF does not differ.

Although of babies born after assisted conception twins have worse perinatal outcomes than singletons, it seems that twins had no increased risk of neurological sequelae as defined in this study. Compared with naturally conceived twins the risk of neurological sequelae in twins after IVF or ICSI was the same, even after exclusion of the monozygotic twins. Children conceived after ICSI carried a similar risk of neurological sequelae as conventional IVF children.

\section{Limitations of the study}

The limitation of this study is the lack of data on the extent to which women in the group of naturally conceived twins had received ovarian stimulation with or without intrauterine insemination (IUI). Our questionnaire study showed that $17.3 \%$ of the control twin mothers conceived after ovarian stimulation with or without IUI. ${ }^{8}$ Since recent papers have reported no influence of the stimulation procedure on neonatal outcome either in singletons or in twins, we assume that the risk of neurological sequelae in children conceived after ovarian stimulation without IVF is no greater than that of children conceived naturally. ${ }^{16}{ }^{17}$

Twins conceived after IVF or ICSI had no greater risk of neurological sequelae, cerebral palsy, or mental retardation than naturally conceived twins or IVF or ICSI singletons. Low birth weight or prematurity as well as male sex independently contributed to the risk of all three outcomes. After adjustment for relevant confounders, twins still had no independent influence on the risk of any of the three outcomes. Hence, being a twin infant per se was no risk factor compared with singletons, and neither was the use of IVF techniques compared with natural conception. In accordance with two recent reports on mental development in ICSI children, we found a similar risk of neurological sequelae and cerebral palsy in ICSI and IVF children. ${ }^{18}{ }^{19}$

As observations on twins are not fully independent, a potential risk exists of estimating the confidence intervals too narrow. When considering our results, the concordance rates were one set of twins in 30 IVF or ICSI children, and six sets in 98 control twins, with neurological sequelae. Although the concordance rates are low, it has to be acknowledged that if one twin is affected then the other is more likely to be affected than would occur by chance.

\section{Strengths of the study}

The strengths of the study were the nationwide design, which allowed us to assess for confounder effects and to evaluate the role of zygosity. The compulsory Danish registry for in vitro fertilisation and the civil registration system enabled us to trace all IVF or ICSI deliveries. A unique linkage between each mother and her children in the medical birth registry allowed us to enrol the children and by cross linkage with the child's identification number from the civil registration system we identified all neurological sequelae diagnosed in a hospital setting.

Moreover, the liberal access to assisted reproductive technologies, including reimbursement of the first three IVF treatments, makes Denmark the country with the highest number of performed IVF cycles per inhabitant in Europe. This is probably the reason why no fundamental differences exist in socioeconomic position between Danish IVF mothers and mothers who conceive spontaneously. This was confirmed in our recent national questionnaire study restricted to children born in 1997.8

\section{Comparison with other studies}

In most existing surveys the follow up period is too short or the sample size insufficient to draw firm conclusions. ${ }^{20}{ }^{21}$ Only one recent Swedish register study on children born from 1982 to 1995 that examined neurological sequelae in children born after assisted conception had a sufficiently large sample size (2060 IVF twins). In this study, IVF twins were compared with a matched group of non-IVF twins, and IVF singletons were compared with a matched group of non-IVF singletons. ${ }^{7}$ The researchers found that IVF twins had a similar risk of neurological sequelae as their naturally conceived peers, but, in contrast to our results, they showed that the risk of neurological sequelae was higher in IVF twins than in IVF singletons. They did not, however, study the effect of ICSI. The crude prevalence of cerebral palsy per 1000 children in the Swedish study was 7.4, 6.9, and 3.8 for IVF/ICSI twins, control twins and IVF or ICSI singletons, respectively. In our study the corresponding rates were 3.2/1000 for IVF or ICSI twins, 4.0/1000 for control twins, and 2.5/1000 for IVF or ICSI singletons. The crude prevalence of cerebral palsy in the total population of the western healthcare region of Sweden in 1991-4 was 2.3/1000. ${ }^{22}$ An overestimation of the prevalence of cerebral palsy in the study of Strömberg et al could have resulted from children being diagnosed by healthcare professionals other than doctors as well as from the shorter follow up period. ${ }^{7}$ Only $25 \%$ of the Swedish children were older than 3 years, at the time of follow up, in con- 
trast to $62 \%$ of the Danish children, and the minimum age of the Swedish children was 18 months, compared with 24 months in the Danish children. The crude prevalence of cerebral palsy in eastern Denmark in 1987-90 was 2.4/1000. ${ }^{23}$ Although this figure is very close to the prevalence found in the IVF or ICSI singletons in our study, it cannot be concluded that the rate of cerebral palsy in these singletons is the same as in the general population, as we relied on diagnoses recorded in a hospital setting. Firstly, not all children with cerebral palsy are treated in hospital, and secondly not all children with cerebral palsy, admitted to hospital for other reasons, will have their diagnosis of cerebral palsy recorded at that time. These problems, however, should not bias either study and do not explain the difference between their results.

\section{Definition of neurological sequelae}

The definition of neurological sequelae and the ranking of diagnoses in our study can also be discussed. To ensure the most objective classification of diagnoses we outlined the ranking system before data retrieval, and to keep a consistent recording of diagnoses we defined neurological sequelae as only the most severe neurological and psychiatric diagnoses. In the Swedish study the primary diagnosis, in case the child had more than one diagnosis, was chosen as the child's major diagnosis from the centre for disabled children. Another reason for the higher Swedish prevalence rates of cerebral palsy could be that the Swedish study comprised an earlier cohort of children born between 1982 and 1995, whereas the children enrolled in our study were born between 1 January 1995 and 31 December 2000. The prenatal and neonatal care of IVF and twin pregnancies have improved during that period.

Our recent published paper on neonatal outcome in twins after assisted conception showed a marginally higher rate of admissions to neonatal intensive care units in IVF or ICSI twins $(56.3 \%)$ than in naturally conceived twins $(52.4 \%)$. No difference was obvious in the risk of caesarean section after stratification for maternal age and parity. ${ }^{9}$ One cannot preclude that the higher admission rate to intensive care in twins after assisted conception has affected the prevalence of neurological sequelae favourably. We assume, however, that this increased rate is due to more precautions being taken in IVF and ICSI twins when considering the borderline cases of whether to admit to intensive care or not. In Denmark no differences exist in the recommendation of prenatal care to IVF compared with naturally conceived twin pregnancies; rather, the dividing line in terms of obstetric care depends on chorionisity. Since monochorionic twins have an increased risk of morbidity and mortality, prenatal care of these twin pregnancies is more intense. This includes more ultrasound examinations to detect intrauterine growth retardation.

\section{Potential biases}

As table 1 shows, infant mortality in control twins was marginally higher than in twins after assisted conception $(\mathrm{P}=0.04)$, mainly because of early neonatal deaths. Hence the prevalence rates of neurological sequelae were not underestimated in favour of IVF or ICSI twins because of higher mortality in these twins. Regarding IVF or ICSI twins versus singletons we observed no significant differences in mortality, and this could not bias the study. Excluding infant deaths from the denominator had negligible influence on the prevalence of neurological sequelae in the three cohorts. Consistent with an earlier survey, we found similar risks of neurological sequelae and cerebral palsy in twins of the same sex compared with twins of the opposite sex. ${ }^{6}$

\section{What is already known on this topic}

Children born after assisted conception have a higher risk of neurological sequelae than naturally conceived children, mainly because of the higher frequency of twins

\section{What this study adds}

Twins born after assisted conception have a similar risk of neurological sequelae as naturally conceived twins and singletons born after assisted conception

The risk of neurological sequelae in children born after ICSI does not differ from that of children born after IVF

\section{Conclusion}

The similar prevalence of neurological sequelae in twins and singletons born after assisted conception we found in our study was reassuring. It should, however, be acknowledged that recent studies have observed higher risks of other adverse outcomes, including a doubled stillbirth rate, all with familiar consequences and higher cost. $.^{2-5} 8-10$ The impact of our study should therefore not be a blind acceptance of dual embryo replacement in IVF but a well considered step towards elective single embryo transfer to the patients who are prone to having twins.

We thank Lone Mortensen, Health Statistics, Danish National Board of Health.

Contributors: AL contributed to study design, interpretation of results, and reviewing the manuscript. LS participated in interpretation of results and reviewing the manuscript. GG supervised planning of classification of outcome and reviewing the manuscript. SR undertook the data retrieval. ANA contributed to study planning, interpretation of results, and reviewing the manuscript. AP was involved in study design, analyses of results, and writing the report. ANA is the guarantor.

Funding: Danish Medical Research Council; Danish Hospital Foundation for Medical Research; Region of Copenhagen, the Faroe Islands and Greenland; and the Research Foundation of Queen Louise's Paediatric Hospital.

Competing interests: None declared.

Ethical approval: The trial was approved by the local research ethics committee and the Danish Data Protection Agency. A specific authorisation from the Danish Data Protection Agency was obtained to data retrieval from the Danish Psychiatric Central Registry.

1 Nyboe Andersen A, Gianaroli L, Nygren KG. Assisted reproductive technology in Europe, 2000. Results generated from European registers by ESHRE. Hum Reprod 2004;19:490-03.

2 Bergh T, Ericson A, Hillensjö T, Nygren KG, Wennerholm UB. Deliveries and children born after in-vitro fertilisation in Sweden 1982-95: a retrospective cohort study. Lancet 1999;354:1579-85

3 Dhont M, Sutter PD, Ruyssinck G, Martens G, Bekaert A. Perinatal outcome of pregnancies after assisted reproduction: a case-control study. Am J Obstet Gynecol 1999;181:688-95.

4 Westergaard HB, Johansen AMT, Erb K, Nyboe Andersen A. Danish national in-vitro fertilization registry 1994 and 1995: a controlled study of birth, malformations and cytogenetic findings. Hum Reprod 1999;14:1896-902.

5 Schieve LA, Meikle SF, Ferre C, Peterson HB, Jeng G, Wilcox LS. Low and very low birth weight in infants conceived with use of assisted reproductive technology. $N$ EnglJ Med 2002.346:731-37.

6 Scher AI, Petterson B, Blair E, Ellenberg JH, Grether JK, Haan E, et al. The Risk of mortality or cerebral palsy in twins: a collaborative population-based study. Pediatr Res 2002;52:671-81

7 Strömberg B, Dahlquist G, Ericson A, Finnström O, Köster M, Stjernquist, K. Neurological sequelae in children born after in-vitro fertilisation: a population based study. Lancet 2002;359:461-65.

8 Pinborg A, Loft A, Schmidt L, Nyboe Andersen A. Morbidity in a Danish national cohort of 472 IVF/ICSI twins, 1132 non-IVF/ICSI twins and 634 IVF/ICSI singletons: health-related and social implications for the children and their families. Hum Reprod 2003;18:1234-43.

9 Pinborg A, Loft A, Rasmussen S, Schmidt L, Langhoff-Roos J, Greisen G, Nyboe Andersen A. Neonatal outcome in a Danish national cohort of 3438 IVF/ICSI twins and 10362 non-IVF/ICSI twins born in 1995 to 2000. Hum Reprod 2004;19:435-41.

10 Pinborg A, Loft A, Nyboe Andersen A. Neonatal outcome in a Danish national cohor of 8602 children born after in vitro fertilization (IVF) or intracytoplasmic sperm injection (ICSI): the role of twin pregnancy. Acta Gynecol Obstet Scand 2004 (in press).

11 Knudsen L, Olsen J. The Danish Medical Birth Registry. Dan Med Bull 1998;45:320-3. 
12 Nyboe Andersen A, Westergaard HB, Olsen J. The Danish in vitro fertilisation (IVF) registry. Dan Med Bull 1999;46:357-60.

13 Andersen TF, Madsen M, Jorgensen J, Mellemkjaer L, Olsen J. The Danish hospital register. A valuable source of data for modern health sciences. Dan Med Bull 1999;46:26368.

14 Munk-Jorgensen P, Mortensen PB. The Danish psychiatric central register. Dan Med Bull 1997;44:82-4

15 Weinberg W. Beiträge zur Physiologie und Pathologie der Mehrlingsgebuhrten beim Menschen [Contributions to physiology and pathology of multiple births in humans]. Archiv Gesamte Physiol Menschen Tiere 1902;88:346-430.

16 Daniel Y, Ochshorn Y, Fait G, Geva E, Bar-Am A, Lessing JB. Analysis of 104 twin pregnancies conceived with assisted reproductive technologies and 193 spontaneously conceived twin pregnancies. Fertil Steril 2000;74:683-89.

17 Källen B, Olausson PO, Nygren KG. Neonatal outcome in pregnancies from ovarian stimulation. Obstet Gynecol, 2002;100:414-9.

18 Sutcliffe AG, Taylor B, Saunders K, Thornton S, Lieberman BA, Grudzinskas JG. Outcome in the second year of life after in-vitro fertilisation by intracytoplasmic sperm injection: a UK case-control study. Lancet 2001;357:2080-84.

19 Leslie GI, Gibson FL, McMahon C, Cohen J, Saunders DM, Tennant C. Children conceived using ICSI do not have an increased risk of delayed mental development at 5 years of age. Hum Reprod 2003;18:2067-72.

20 Minakami H, Sayama M, Honma Y, Matsubara S, Koike T, Sato I, et al. Lower risk of adverse outcome in twins conceived by artificial reproductive techniques compared with spontaneously conceived twins. Hum Reprod 1998;13:2005-8.

21 Koivurova S, Hartikainen AL, Sovio U, Gissler M, Hemminki E, Järvelin MR. Growth, psychomotor development and morbidity up to 3 years of age in children born after IVF. Hum Reprod 2003;18:2328-36.
22 Hagberg B, Hagberg G, Beckung E, Uvebrandt P. Changing panorama of cerebral palsy in Sweden. VIII. Prevalence and origin in the birth year period 1991-94. Acta Paediat 2001;90:271-7.

23 Topp M, Uldall P, Greisen G. Cerebral palsy births in eastern Denmark, 1987-1990: implications for neonatal care. Pediatr Perinat Epidemiol 2001;15:271-7.

(Accepted 3 June 2004)

doi 10.1136/bmj.38156.715694.3A

Fertility Clinic, University of Copenhagen, Rigshospitalet, Blegdamsvej 9, DK-2100 Copenhagen, Denmark

Anja Pinborg research fellow

Anne Loft consultant

Anders Nyboe Andersen professor

Institute of Public Health, University of Copenhagen, Blegdamsvej 3, DK-2200

Copenhagen, Denmark

Lone Schmidt associate professor

Department of Neonatology, University of Copenhagen, Rigshospitalet

Gorm Greisen professor

National Board of Health, Health Statistics, Islandsbrygge 67, DK-2300

Copenhagen, Denmark

Steen Rasmussen senior consultant

Correspondence to: A Pinborg apinborg@rh.dk 